\title{
Social Interaction with Cultural Heritage on the Web*
}

\author{
Max Arends and Doron Goldfarb \\ Institut für Softwaretechnik und Interaktive Systeme \\ Technische Universität Wien \\ Favoritenstraße 9-11, A-1040 Wien
}

\begin{abstract}
This research proposal aims at bridging some of the current trends regarding Cultural Heritage institution's usage of Web based technologies. These trends include the increased use of Social Web principles with respect to traditional Web presences and the employment of Web3D technologies for single- or multi-user 3D environments for entertainment and education. Seen individually, each of these topics has already been covered by a number of different research projects, but relatively little work has been done on finding ways to elaborate these approaches by combining them within a common framework. The latter can also be observed in the practical usage patterns of these technologies, being usually limited to rather self-contained projects. Therefore, the aim is to provide a framework for the presentation of cultural heritage artefacts in a $3 \mathrm{D}$ virtual collaborative environment where visitors are able to participate using both synchronous and asynchronous means of communication.
\end{abstract}

\section{Introduction}

Cultural Heritage Institutions nowadays need far more advanced Web presentations in order to get in touch with their potential visitors. It's not sufficient anymore to have a Web page that informs visitors only about basic information like opening hours and the current exhibition. Since the Web 2.0 era, visitors are much more involved in the contribution of information, and appreciate the information contributed by others. This can also be helpful for different groups of visitors, as people with a novice knowledge use a different jargon than persons with an expert knowledge of Cultural Heritage information. With the use of user-contributed tags for artefacts, this gap may be closed. As Chan observes "these user keywords most often are generally descriptive, allowing users to discover objects that are difficult to discover through the $\mathrm{Mu}-$ seum's formal classification system" [3]. Cultural Heritage Institutions nowadays also aim to contribute their whole collections on their Web site and to provide an idea of their cultural goal. This is where a $3 \mathrm{D}$ environment has advantages to traditional viewing. In a study presented in [1] two groups of students were presented with either a paper reproduction of an artefact or a 3D representation, and then asked to come up with an appropriate title of the artefact. Participants of the 3D group tended to "think not 'what' they faced, but to 'why' or 'how' something was in front of them". If this is combined with multi-user capabilities, so called Multi-User Virtual Environments

* Supervised by Dieter Merkl, PhD, Associate Professor. 
(MUVE), where multiple users are able to interact with each other simultaneously, the benefits of imparting cultural heritage information shall increase even further. Therefore the development of a multi-user 3D environment featuring social-web functionality might prove beneficial for both, the institutions and their visitors.

In our expectation, users of our 3D MUVE will especially gain a better overview of the cultural context the presented objects are embedded in, as for example the knowledge about relationships between artists, their oeuvre and general historical information is important for the understanding of individual artworks.

\section{Research Objectives}

\subsection{Find Useful Visualisation Approaches}

The spectrum of visualisation methods spans from real-life simulation [5] to completely abstract Information Visualisation metaphors [8]. Key elements of Cultural Heritage related presentations are the visualisation of the artefacts themselves, their respective cross-references to other objects, concepts or people according to specific attributes like creator, style or location. The environment in which all the information is presented plays an important role as well. Given the huge variety of Cultural Heritage content, it cannot be expected to find a single visualisation approach that satisfies all the different requirements. Therefore, a number of experiments should be carried out in order to find suitable methods for well-defined usage scenarios, e.g. to find the best way of displaying relationships between different painters and how these relations might have influenced their work.

\subsection{Derive New Cross-References and Classifications through User Contribution}

Cross-references between artefacts and their respective classification schemes are usually derived from expert knowledge. As Web 2.0-style user contributions like tagging, rating or commenting may lay the foundation of user-generated indexes - as for example discussed in [3] - that might be easier to comprehend for larger audiences, their integration into the environment is another dedicated objective. This raises issues regarding the interfaces that provide the ability to contribute, as well as how participation can be fostered.

\subsection{Analyze Usage Patterns}

Since user's behaviour in virtual environments can be tracked much more easily than in real life, valuable information regarding the visitor's interests and interaction patterns can be collected. The respective findings can be used by Cultural Heritage institutions to better understanding their audiences, potentially leading to increased benefit for both sides. An interesting example where visitor behaviour is studied can be found in [4].

\subsection{Construct Narratives}

Simply visualising content organised according to a number of common attributes might be perceived as too artificial and lacking consistent storytelling. "Hard coding" 
of narratives would, however, take away much of the flexibility of virtual presentation systems. Therefore, mechanisms should be included that enable domain experts and interested visitors to create "stories" about the presented content, e.g. to organise a collection of artefacts according to specific attributes and to put them into a chronological sequence that might be more based on subjective criteria than on hard facts only. This can only be achieved by providing some kind of "high-level" language that is dedicated to such storytelling information. Examples for such languages are proposed in [9] and in [6].

\subsection{Harvesting Web Based Data Repositories}

Cultural Heritage data is usually stored in proprietary formats, dependent on the respective institution's policy. Throughout the recent years, however, more and more data repositories are opened up to the public and organized according to common standards and technologies. Providing interfaces to these open data collections would therefore enable to overcome institutional boundaries and facilitate the linkage of existing information sources by combining them to "Mash-Ups" within the 3D environment.

\section{Research Methodology}

Get an overview of Cultural Heritage Web institutions use of Web based technologies. The websites of cultural heritage institutions are analysed according to their web features.

Overview of Web 3D in Cultural Heritage. Different Web3D solutions are examined in order to get an idea of how cultural heritage is represented in virtual environments.

Find a suitable platform. Different multi-user virtual 3D environments like Second Life, OpenSim, Project Wonderland and Unity3D are evaluated according to openness, scalability, data integration and performance.

Consider different approaches for the internal representation of Cultural Heritage information. A data model is developed that is scalable of good performance to represent large amounts of cultural heritage information by studying what representation models are available for cultural heritage and how thesauri and web data-sources may be used in order to connect different sources.

Consider different visualisation metaphors for different audiences. Different skills of visitors like school children, adults or experts need different visualisation metaphors in order put across the information. Therefore an open system is developed, where different skilled visitors are able to collaboratively use the system.

The elaborated methods are implemented in a prototype.

Evaluation. The prototype is evaluated by testers with different levels of computer skills and knowledge of Cultural Heritage information. Evaluation should focus on both improving the learning outcome as well as improving the overall usability. We intend this process to iterate between the steps of finding visualisation metaphors, implementation and evaluating their effect. 


\section{Current Status}

Until this moment we are working on the implementation of our first prototype. We started by getting an overview of the way that Cultural Heritage Institutions use Web sites and community-oriented Web 2.0 features in order to communicate with their potential visitors. This was done by analysing the websites of 69 art museums from all over the world, including the 20 most visited, according to their collaborative and interactive aspects. Our results were presented at the IADIS 2009 and published in [2].

By checking the use of Web 3D in cultural heritage institutions, it was interesting to see that there are two different kinds of approaches. The first is to create a realistic replica of a museum in a 3D virtual environment, as done by the Old Masters Picture Gallery of the Dresden State Art Collections [5], where the whole facade including all rooms and artefacts are modelled true-to-scale in Second Life. The other approach is more abstract, where information is thematically arranged and it is possible to arrange and compare artefacts according to their artists, sujets, era, geographical information, etc.

We were then looking for a suitable platform that combines openness, free availability for academic use and the possibility to run the application as a browser-plugin. After looking at Second Life, OpenSim and Project Wonderland, we decided to use the GameEngine Unity3D, which comes with a browser-plugin that allows users to access the application directly with their browser and not having to install and run a stand-alone application on their computer.

In order to develop a data-model that offers scalability and is suited for the use of cultural heritage information, we first chose to use the CIDOC CRM, "a formal ontology intended to facilitate the integration, mediation and interchange of heterogeneous cultural heritage information" [7], but later on decided to follow the VRA Core based approach introduced in [10]. In order to improve the access to the information for users, we integrated the Getty Thesaurus of Geographic Names (TGN) and the Union List of Artist Names (ULAN) into our data-model.

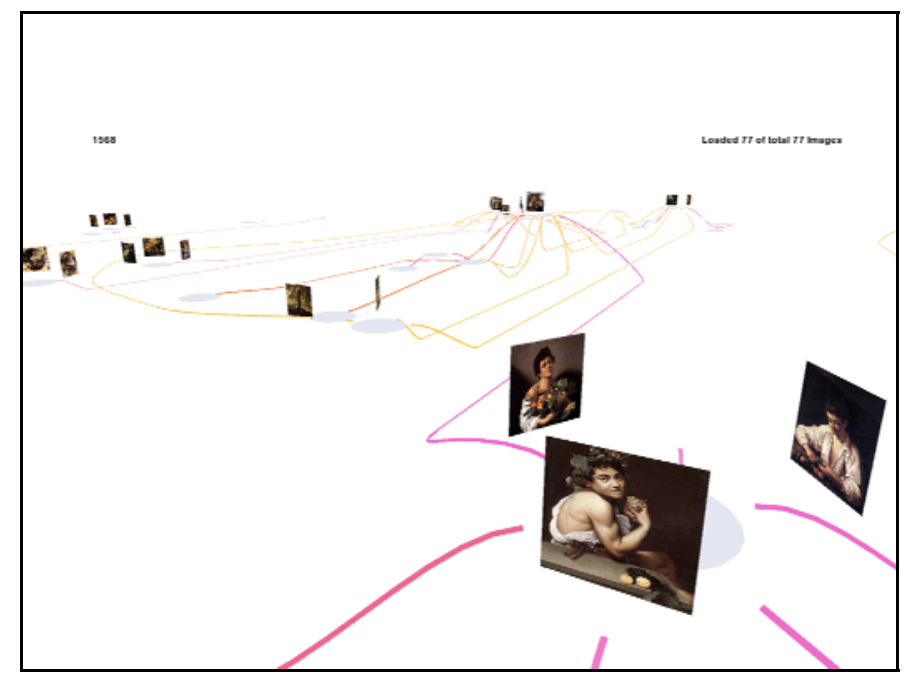

Fig. 1. Information Landscape displaying artists and their work, interconnected by lines representing their different types of relationships 


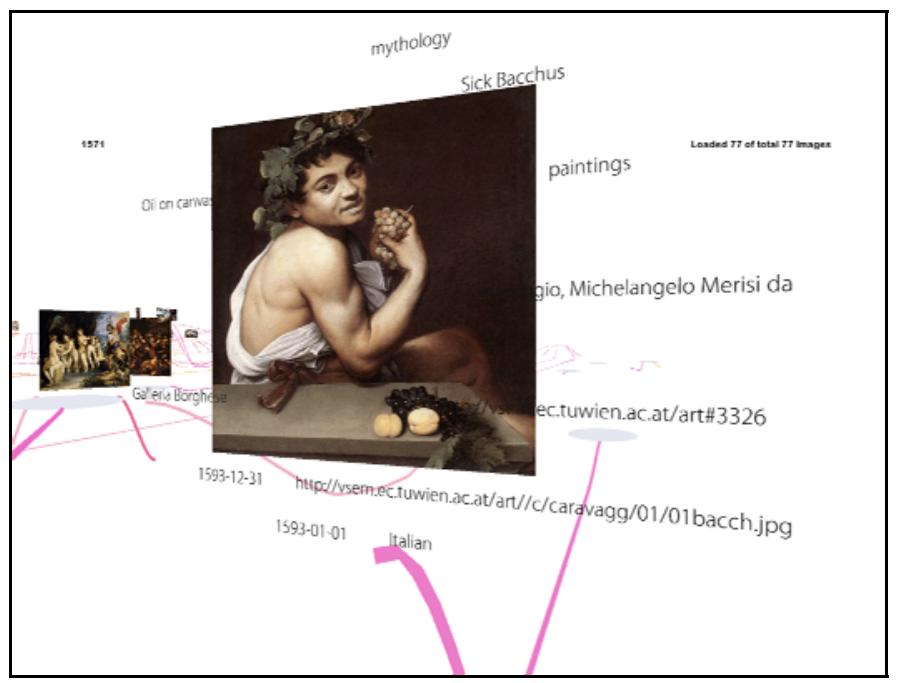

Fig. 2. Close up of an artwork. Existing metadata is displayed around the virtual canvas.

We are currently working on the development of our first prototype, getting familiar with Unity3D and implement different narratives for different groups of users.

The following figures should give an impression of our current environment. Figure 1 shows an overview on the Information Landscape that is created from relationships between artists from the Getty ULAN database. Each artist is represented as a node and is surrounded by his or her artworks. The differently coloured edges between the nodes show known relations between artists, each color represents a certain type of relationship like teacher/student, patronage etc. In Figure 2, a close up of an artwork is shown. Metadata describing the title, date of creation, type and sujet etc. is displayed when the virtual visitor gets close to the artifact.

\section{Main Contribution}

The main contribution of this work to the field of web engineering consists of providing a Web3D based environment for browsing and querying Cultural Heritage content that is available on the Web. Finding methods to organise and present "Mash-Ups" of existing data sources in a dynamic Multi-User 3D environment might provide new insight about existing relationships and more intuitive ways of interaction with Web content.

\section{Acknowledgment}

This work is funded by the FWF (Fonds zur Förderung der wissenschaftlichen Forschung / Austrian Science Fund), Project No. L602, "The Virtual 3D Social Experience Museum". 


\section{References}

[1] Antonietti, A., Cantoia, M.: To see a painting versus to walk in a painting: an experiment on sense-making through virtual reality. Computers \& Education 34(3-4), 213-223 (2000)

[2] Arends, M., Goldfarb, D., Merkl, W., Weingartner, M.: Interaction With Art Museums on the Web. In: Isaias, P., White, B., Nunes, M.B. (eds.) Proceedings of the IADIS Int'l. Conference WWW/Internet 2009. IADIS Press (2009)

[3] Chan, S.: Tagging and searching - Serendipity and museum collection databases. In: Proceedings of the Museums and the Web Conference, San Francisco, CA (2007)

[4] Cosley, D., Baxter, J., Lee, S., Alson, B., Nomura, S., Adams, P., Sarabu, C., Gay, G.: A tag in the hand: supporting semantic, social, and spatial navigation in museums. In: Proceedings of the 27th International Conference on Human Factors in Computing Systems, Boston, MA, USA (2009)

[5] Dresden Gallery, http: / / www . dresdengallery.com

[6] Not, E., Callaway, C., Rocchi, C., Stock, O., Zancanaro, M.: Cinematographic techniques for automatic documentary-like presentations. In: Stock, O., Zancanaro, M. (eds.) PEACH: Intelligent Interfaces for Museum Visits. Cognitive Technologies Series, pp. 23-44. Springer, Berlin (2007)

[7] Past and Future of ISO21127:2006 or CIDOC CRM, http://cidoc.mediahost.org/standard_crm(en) (E1).xml (2006)

[8] Ruffaldi, E., Evangelista, C., Neri, V., Carrozzino, M., Bergamasco, M.: Design of information landscapes for cultural heritage content. In: Proceedings of the 3rd International Conference on Digital Interactive Media in Entertainment and Arts, Athens, Greece, pp. 113-119 (2008)

[9] TourML specification, http://wiki.museummobile.info/museums-togo/productsservices/tourml

[10] Van Assem, M., Van Ossenbruggen, J., Schreiber, G.: The VRA Core Application Profile for searching and presenting cultural heritage: the MultimediaN case. In: Proceedings of the International Conference on Dublin Core and Metadata Applications, Pittsburgh, PA, USA (2010) 